Pacific Journal of Mathematics

A FORMULA FOR THE NORMAL PART OF THE
LAPLACE-BELTRAMI OPERATOR ON THE FOLIATED

ARA AND SHINSUKE YOROZU 


\section{A FORMULA FOR THE NORMAL PART OF THE LAPLACE-BELTRAMI OPERATOR ON THE FOLIATED MANIFOLD}

\section{Haruo Kitahara and Shinsuke YorozU}

In this paper, we give a formula for the normal part of the Laplace-Beltrami operator with respect to the second connection on a foliated manifold with a bundle-like metric. This formula is analogous to the formula obtained by $\mathrm{S}$. Helgason.

1. Itroduction. We shall be in $C^{\infty}$-category and manifolds are supposed to be paracompact, connected Hausdorff spaces.

Let $M$ be a complete $(p+q)$-dimensional Riemannian manifold and $H$ a compact subgroup of the Lie group of all isometries of $M$. We suppose that all orbits of $H$ have the same dimension $p$. Then $H$ defines a $p$-dimensional foliation $F$ whose leaves are orbits of $H$, and the Riemannian metric is a bundle-like metric with respect to the foliation $F$. A quotient space $B=M / F$ is a Riemannian $V$-manifold [5]. Let $L_{D}$ be the Laplace-Beltrami operator on $M$ with respect to the second connection $D[8]$, and let $\Delta\left(L_{D}\right)$ denote the operator defined by $\left({ }^{*}\right)$ in $\S 4$. Our goal in this paper is the following theorem:

THEOREM. Let $L_{D}$ be the Laplace-Beltrami operator on $M$ with respect to the second connection $D$ and $L_{B}$ the Laplace-Beltrami operator on $B$ with respect to the Levi-Civita connection associated with the Riemannian metric defined by the normal component of the metric on $M$. Then

$$
\Delta\left(L_{D}\right)=\delta^{-1 / 2} L_{B} \circ \delta^{1 / 2}-\delta^{-1 / 2} L_{B}\left(\delta^{1 / 2}\right)
$$

where $\delta$ is the function given by $\left(^{* *}\right)$ below.

This theorem is analogous to the following result obtained by S. Helgason [2]: Suppose $V$ is a Riemannian manifold, $H$ a closed unimodular subgroup of the Lie group of all isometries of $V$ (with the compact open topology). Let $W \subset V$ be a submanifold satisfying the condition: For each $w \in W$,

$$
(H \cdot w) \cap W=\{w\}, \quad V_{w}=(H \cdot w)_{w} \oplus W_{w},
$$

where $\oplus$ denotes orthogonal direct sum. Let $L_{V}$ and $L_{W}$ denote the Laplace-Beltrami operators on $V$ and $W$, respectively. Then

$$
\Delta\left(L_{V}\right)=\delta^{-1 / 2} L_{W} \circ \delta^{1 / 2}-\delta^{-1 / 2} L_{W}\left(\delta^{1 / 2}\right)
$$


where $\Delta\left(L_{V}\right)$ denotes the operator called the radial part of $L_{V}$ and $\delta$ is the function given by $d \sigma_{w}=\delta(w) d h\left(d \sigma_{W}\right.$ is the Riemannian volume element on the orbit $H \cdot w$ and $d \dot{h}$ is an $H$-invariant measure on each orbit $H \cdot w=H /\{$ the isotropy subgroup of $H$ at $w\}$ ).

2. Definition of $V$-manifold $[1,6,7]$. The concept of $V$-manifold is defined by I. Satake. Let $M$ be a Hausdorff space. $A C^{\infty}$ local uniformizing system $\{\tilde{U}, G, \varphi\}$ for an open set $U$ in $M$ is a collection of the following objects:

$\widetilde{U}$ : a connected open set in the $m$-dimensional Euclidean space (or $C^{\infty}$-manifold).

$G$ : a finite group of $C^{\infty}$-transformations of $\tilde{U}$.

$\varphi$ : a continuous map from $\widetilde{U}$ onto $U$ such that $\varphi \circ \sigma=\varphi$ for all $\sigma \in G$, inducing a homeomorphism from the quotient space $\tilde{U} / G$ onto $U$.

Let $\{\widetilde{U}, G, \varphi\},\left\{\tilde{U}^{\prime}, G^{\prime}, \varphi^{\prime}\right\}$ be local uniformizing systems for $U$, $U^{\prime}$ respectively, and let $U \subset U^{\prime}$. By a $C^{\infty}$-injection $\lambda:\{\tilde{U}, G, \varphi\} \rightarrow$ $\left\{\widetilde{U}^{\prime}, G^{\prime}, \varphi^{\prime}\right\}$ we mean a $C^{\infty}$-isomorphism from $\widetilde{U}$ onto an open subset of $\widetilde{U}^{\prime}$ such that for any $\sigma \in G$ there exists $\sigma^{\prime} \in G^{\prime}$ satisfying relations $\varphi=\varphi^{\prime} \circ \lambda$ and $\lambda \circ \sigma=\sigma^{\prime} \circ \lambda$.

A $C^{\infty}-V$-manifold consists of a connected Hausdorff space $M$ and a family $\mathscr{F}$ of $C^{\infty}$-local uniformizing systems for open subsets in $M$ satisfying the following conditions:

(I) If $\{\tilde{U}, G, \varphi\},\left\{\tilde{U}^{\prime}, G^{\prime}, \varphi^{\prime}\right\} \in \mathscr{F}$ and $U \subset U^{\prime}$, then there exists a $C^{\infty}$-injection $\lambda:\{\widetilde{U}, G, \varphi\} \rightarrow\left\{\widetilde{U}^{\prime}, G^{\prime}, \varphi^{\prime}\right\}$.

(II) The open sets $U$, for which there exists a local uniformizing system $\{\tilde{U}, G, \varphi\} \in \mathscr{F}$, form a basis of open sets in $M$.

The set $R$ of all real numbers is regarded as a $V$-manifold defined by a single local uniformizing system $\{R,\{1\}, 1\}$, then a $C^{\infty}$-function on a $V$-manifold $(M, \mathscr{F})$ is defined as a $C^{\infty}$-map $M \rightarrow R$ defined by a $C^{\infty}-V$-manifold map $(M, \mathscr{F}) \rightarrow(R,\{R,\{1\}, 1\})$.

A $C^{\infty}-V$-bundle over $C^{\infty}-V$-manifold is also defined, and in particular the tangent bundle $\left(T M, \mathscr{F}^{*}\right)$ of a $C^{\infty}-V$-manifold $(M, \mathscr{F})$ is defined. Let $(M, \mathscr{F})$ be a $C^{\infty}-V$-manifold, then an $h$-form $\omega$ on $(M, \mathscr{F})$ is a collection of $h$-forms $\left\{\omega_{\widetilde{U}}\right\}$, where $\omega_{\widetilde{U}}$ is a $G$-invariant $h$-form on $\widetilde{U}$ such that $\omega_{\tilde{U}}=\omega_{\tilde{U}}, \circ \lambda$ for any injection $\lambda:\{\tilde{U}, G, \varphi\} \rightarrow$ $\left\{\widetilde{U}^{\prime}, G^{\prime}, \varphi^{\prime}\right\}\left(\{\widetilde{U}, G, \varphi\},\left\{\widetilde{U}^{\prime}, G^{\prime}, \varphi^{\prime}\right\} \in \mathscr{F}\right)$, and if the support of $\omega$ is contained in $U=\varphi(\tilde{U})$,

$$
\int_{M} \omega:=\frac{1}{N_{G}} \int_{\widetilde{U}} \omega_{\widetilde{U}}
$$

where $N_{G}$ denotes the order of $G$. A Riemannian metric $g$ on $(M$, $\mathscr{F})$ is a collection of Riemannian metrices $\left\{g_{\tilde{U}}\right\}$, where $g_{\tilde{U}}$ is a $G$ invariant Riemannian metric on $\tilde{U}$ satisfying some condition with 
any injection $\lambda:\{\tilde{U}, G, \varphi\} \rightarrow\left\{\tilde{U}^{\prime}, G^{\prime}, \varphi^{\prime}\right\}$.

3. Review of the results from $[4,5]$. Let $M$ be a complete $(p+q)$-dimensional manifold with a "bundle-like matric" with respect to a $p$-dimensional foliation $F$. We suppose that each leaf of the foliation $F$ is closed.

The quotient space $B=M / F$ is the space formed from $M$ by identifying each leaf to a point, and let $\pi: M \rightarrow B$ denote the identification map. $H(S)$ denotes the holonomy group of a leaf $S$. Since $M$ has the bundle-like metric with respect to $F$ and all leaves are closed, $H(S)$ is a finite group for any $S$ and $B$ is a metric space defining the distance between two points of $B$ to be the minimum distance between them considered as leaves is $M . \quad B$ is a connected Hausdorff space, since it is metric space and is the continuous image of $M$ under $\pi$. Given any point $b \in B$, let $S=\pi^{-1}(b)$. Let $U$ be a flat coordinate neighborhood of some point of $S$. Since $H(S)$ may be considered as a group of isometries of the sphere of unit vectors orthogonal to the leaf $S$ at some arbitrary point of $S, H(S)$ operates the $q$-ball orthogonal to $S$. Thus we may consider that $H(S)$ operates on $U$ such a manner that $\{U, H(S), \pi\}$ is a local uniformizing system for the neighborhood $\pi(U)$ in $B$. The natural injection map of two such local uniformizing systems are of $C^{\infty}$. Thus $B$ is a $C^{\infty}-V$-manifold. Since $H(S)$ is an isometry on the normal vectors at a point of $S$, the normal component of the metric of $M$ defines a Riemannian structure on $B$. Thus $B$ is a Riemannian $V$-manifold.

4. Laplace-Beltrami operator with respect to the second connection. Let $M$ be a $(p+q)$-dimensional manifold with a Riemannian metric $\langle$,$\rangle and a p$-dimensional foliation $F$. Let $\left(U,\left(x^{1}\right.\right.$, $\left.\cdots, x^{p}, y^{1}, \cdots, y^{p}\right)$ ) be a flat coordinate neighborhood system, that is, in $U$, the foliation $F$ is defined by $d y^{\alpha}=0$ for $1 \leqq \alpha \leqq q$. Hereafter we will agree on the following ranges of indices: $1 \leqq i, j$, $k \leqq p, 1 \leqq \alpha, \beta, \gamma, \delta \leqq q$.

We may choose in each flat coordinate neighborhood system $\left(U,\left(x^{1}, \cdots, x^{p}, y^{1}, \cdots, y^{q}\right)\right) 1$-forms $w^{1}, \cdots, w^{p}$ such that $\left\{w^{1}, \cdots, w^{p}\right.$, $\left.d y^{1}, \cdots, d y^{q}\right\}$ is a basis for the cotangent space, and vectors $v_{1}, \cdots$, $v_{q}$ such that $\left\{\partial / \partial x^{1}, \cdots, \partial / \partial x^{p}, v_{1}, \cdots, v_{q}\right\}$ is the dual base for the tangent space. Then we may get

$$
w^{i}:=d x^{i}+A_{\alpha}^{i} d y^{\alpha}, \quad v_{\alpha}:=\frac{\partial}{\partial y^{\alpha}}-A_{\alpha}^{i} \frac{\partial}{\partial x^{i}} .
$$

We may choose $A_{\alpha}^{i}$ such that $\left\langle\partial / \partial x^{i}, v_{\alpha}\right\rangle=0$, then the metric has the local expression 


$$
d s^{2}=g_{i j}(x, y) w^{i} w^{j}+g_{\alpha \beta}(x, y) d y^{\alpha} d y^{\beta}
$$

where

$$
g_{i j}:=\left\langle\frac{\partial}{\partial x^{i}}, \frac{\partial}{\partial x^{j}}\right\rangle, \quad g_{\alpha \beta}:=\left\langle v_{\alpha}, v_{\beta}\right\rangle
$$

and $x:=\left(x^{1}, \cdots, x^{p}\right), y:=\left(y^{1}, \cdots, y^{q}\right)$.

We may uniquely define the "second connection" $D$ on $M$ as follows (cf. [8]);

$$
\begin{aligned}
& D_{\partial / \partial x^{i}} \frac{\partial}{\partial x^{j}}=\Gamma_{j i}^{k} \frac{\partial}{\partial x^{k}}, \quad D_{v_{\alpha}} \frac{\partial}{\partial x^{j}}=\Gamma_{\alpha j}^{k} \frac{\partial}{\partial x^{k}}, \\
& D_{\partial / \partial x^{i}} v_{\beta}=\Gamma_{i \xi}^{\gamma} v_{\gamma}, \quad D_{v_{\alpha}} v_{\beta}=\Gamma_{\alpha \beta}^{\gamma} v_{\gamma},
\end{aligned}
$$

$$
\begin{gathered}
\frac{\partial}{\partial x^{i}}\left\langle\frac{\partial}{\partial x^{j}}, \frac{\partial}{\partial x^{k}}\right\rangle=\left\langle D_{\partial / \partial x^{i}} \frac{\partial}{\partial x^{j}}, \frac{\partial}{\partial x^{k}}\right\rangle+\left\langle\frac{\partial}{\partial x^{\gamma}}, D_{\partial / \partial x^{i}} \frac{\partial}{\partial x^{k}}\right\rangle, \\
v_{\alpha}\left\langle v_{\beta}, v_{\gamma}\right\rangle=\left\langle D_{v_{\alpha}} v_{\beta}, v_{\gamma}\right\rangle+\left\langle v_{\beta}, D_{v_{\alpha}} v_{\gamma}\right\rangle, \\
T\left(\frac{\partial}{\partial x^{i}}, \frac{\partial}{\partial x^{j}}\right)=T_{i j}^{\gamma} v_{\gamma}, \quad T\left(\frac{\partial}{\partial x^{i}}, v_{\beta}\right)=0, \\
T\left(v_{\alpha}, \frac{\partial}{\partial x^{j}}\right)=0, \quad T\left(v_{\alpha}, v_{\beta}\right)=T_{\alpha \beta}^{k} \frac{\partial}{\partial x^{k}},
\end{gathered}
$$

where $T$ denotes the torsion of $D$, that is, for any vector fields $X$, $Y$ on $M, T(X, Y):=D_{X} Y-D_{Y} X-[X, Y]$ ([ , ] denotes the usual bracket operator). Note that, in general, the torsion of $D$ doesn't vanish. If the metric has the local expression

$$
d s^{2}=g_{i j}(x, y) w^{i} w^{j}+g_{\alpha \beta}(y) d y^{\alpha} d y^{\beta},
$$

the metric is called a "bundle-like metric" with respect to the foliation $F$. Hereafter we suppose that $M$ has a bundle-like metric with respect to $F$. Then we get

$$
\frac{\partial}{\partial x^{i}}\left\langle v_{\alpha}, v_{\beta}\right\rangle=\left\langle D_{\partial / \partial x^{i}} v_{\alpha}, v_{\beta}\right\rangle+\left\langle v_{\alpha}, D_{\partial / \partial x^{i}} v_{\beta}\right\rangle .
$$

For a vector field $X$ on $M$, $\operatorname{div}_{D} X$ is defined by

$$
\operatorname{div}_{D} X:=\operatorname{Trace}\left(Y \longrightarrow D_{Y} X\right),
$$

for any vector field $Y$ on $M$. For a function $f$ on $M$, $\operatorname{grad}_{D} f$ is defined by

$$
\begin{aligned}
\operatorname{grad}_{D} f: & =\left(\widetilde{g}^{i j} D_{\partial / \partial x^{j}} f\right) \frac{\partial}{\partial x^{i}}+\left(\widetilde{g}^{\alpha \beta} D_{v_{\beta}} f\right) v_{\alpha} \\
& =\left(\widetilde{g}^{i j} \frac{\partial}{\partial x^{j}}(f)\right) \frac{\partial}{\partial x^{i}}+\left(\widetilde{g}^{\alpha \beta} v_{\beta}(f)\right) v_{\alpha}
\end{aligned}
$$


where $\left(\widetilde{g}^{i j}\right)$ and $\left(\widetilde{g}^{\alpha \beta}\right)$ are inverse matrices of $\left(g_{i j}\right)$ and $\left(g_{\alpha \beta}\right)$ respectively. We define the Laplace-Beltrami operator $L_{D}$ with respect to the second connection $D$ by

$$
L_{D}(f):=\operatorname{div}_{D} \operatorname{grad}_{D} f,
$$

that is,

$$
\begin{aligned}
L_{D}(f)= & \widetilde{g}^{i j} \frac{\partial}{\partial x^{i}}\left(\frac{\partial}{\partial x_{j}}(f)\right)-\widetilde{g}^{i j} \Gamma_{i j}^{k} \frac{\partial}{\partial x^{k}}(f) \\
& +\widetilde{g}^{\alpha \beta} v_{\alpha}\left(v_{\beta}(f)\right)-\widetilde{g}^{\alpha \beta} \Gamma_{\alpha \beta}^{\gamma} v_{\gamma}(f) .
\end{aligned}
$$

Let $B$ be the $C^{\infty}-V$-manifold $M / F$. Let $\mathscr{E}(B)$ (resp. $\mathscr{D}(B)$ be the space of $C^{\infty}$-functions (resp. $C^{\infty}$-functions of compact support) on $B$, and let $\mathscr{E}_{s}(M)$ be the space of $C^{\infty}$-functions on $M$ which are constants on leaves. We may define a map $\Phi: \mathscr{E}_{s}(M) \rightarrow \mathscr{E}(B)$ by $\Phi(f)(\pi(m)):=f(m)$ where $f \in \mathscr{E}_{s}(M), m \in M$ and $\pi: M \rightarrow B$, then $\Phi$ is of one-to-one. Let $\mathscr{E}_{S}^{0}(M):=\Phi^{-1}(\mathscr{D}(B))$.

It is clear that $f \in \mathscr{E}_{s}(M)$ if and only if $\partial / \partial x^{i}(f)=0$ for $1 \leqq i \leqq p$.

LEMma. If $f \in \mathscr{E}_{s}(M)$, then $L_{D}(f) \in \mathscr{E}_{s}(M)$.

Proof. For $f \in \mathscr{E}_{s}(M)$, we get

$$
L_{D}(f)=\widetilde{g}^{\alpha \beta} v_{\alpha}\left(v_{\beta}(f)\right)-\widetilde{g}^{\alpha \beta} \Gamma_{\alpha \beta}^{\gamma} v_{\gamma}(f) .
$$

Since $g_{\alpha \beta}=g_{\alpha \beta}(y)$ and $\Gamma_{\alpha \beta}^{\gamma}=(1 / 2) \widetilde{g}^{\gamma \delta}\left\{v_{\alpha}\left(g_{\delta \beta}\right)+v_{\beta}\left(g_{\alpha \delta}\right)-v_{\delta}\left(g_{\alpha \beta}\right)\right\}$, we get $\widetilde{g}^{\alpha \beta}=\widetilde{g}^{\alpha \beta}(y)$ and so $\partial / \partial x^{i}\left(L_{D}(f)\right)=0$. Thus we get $L_{D}(f) \in \mathscr{E}{ }_{s}(M)$.

REMARK. Let $L$ be the Laplace-Beltrami operator with respect to the Levi-Civita connection associated with the bundle-like metric. In general $L(f) \notin \mathscr{E}_{S}(M)$ for $f \in \mathscr{E}_{S}(M)$.

For $L_{D}$ and $\underline{f} \in \mathscr{E}(B)$, we define $\Delta\left(L_{D}\right)$ by

$$
\Delta\left(L_{D}\right)(\underline{f})(b):=L_{D}\left(\Phi^{-1}(\underline{f})\right)\left(\pi^{-}(b)\right), \quad b \in B .
$$

This is well-defined by lemma. Roughly speaking, $\Delta\left(L_{D}\right)$ seems to be an operator projected on $B$ of the normal part of $L_{D}$.

5. Proof of theorem. Using the same notations as above sections, we give a proof of our theorem.

The isotropy subgroup $H_{m}$ at each point $m \in M$ is compact and the orbit $H \cdot m$ is compact. We fix a Haar measure on $H$ and a Haar measure on $H_{m}$, we get an $H$-invariant measure $d \dot{h}$ on each orbit $H \cdot m=H / H_{m}$. Since $M$ has the bundle-like metric, $d s^{2}=$ $g_{i j}(x, y) w^{i} w^{j}+g_{\alpha \beta}(y) d y^{\alpha} d y^{\beta}$, the volume element $d M$ of $M$ is given by 


$$
\begin{aligned}
d M & =G(x, y) d x^{1} \wedge \cdots \wedge d x^{p} \wedge d y^{1} \wedge \cdots \wedge d y^{q} \\
( & \left.=G(x, y) w^{1} \wedge \cdots \wedge w^{p} \wedge d y^{1} \wedge \cdots \wedge d y^{q}\right)
\end{aligned}
$$

where

$$
G(x, y):=\sqrt{\operatorname{det}\left(\begin{array}{ll}
g_{2} & j 0 \\
0 & g_{\alpha \beta}
\end{array}\right)} .
$$

For a flat coordinate system $\left(U,\left(x^{1}, \cdots, x^{p}, y^{1}, \cdots, y^{q}\right)\right)$ and the projection $\pi: M \rightarrow B$,

$$
d \sigma=G^{\prime}(y) d y^{1} \wedge \cdots \wedge d y^{q},
$$

where $G^{\prime}(y):=\sqrt{\left|\operatorname{det}\left(g_{\alpha \beta}\right)\right|}$, is regarded as the volume element $d B$ of $B$, since $\{U, H(S), \pi\}$ is a local uniformizing system for $\pi(U)$ in $B$. Also we get

$$
G(x, y)=\sqrt{\mid \operatorname{det}\left(g_{i j}(x, y)\right)} \cdot G^{\prime}(y) .
$$

However

$$
\sqrt{\left|\operatorname{det}\left(g_{i j}(x, y)\right)\right|} w^{1} \wedge \cdots \wedge w^{p}
$$

is the volume element $d S_{m}$ on the leaf $S_{m}$ through a point $m=(x, y)$ (that is, on the orbit $H \cdot m$ ). Thus, if $f \in \mathscr{E}_{S}^{\circ}(M)$ we get from the Fubini's theorem that

$$
\int_{M} f d M=\int_{B}\left[\int_{H \cdot m} f d S_{m}\right] d B(\pi(m))
$$

where " " denotes the image under $\Phi . d S_{m}$ is invariant under $H$, so it must be a scalar multiple of $d \dot{h}$,

$$
d S_{m}=\bar{\delta}(m) d \dot{h} .
$$

Then the function $\bar{\delta}$ belongs to $\mathscr{E}_{s}(M)$. We put

$$
\delta:=\Phi(\bar{\delta}) .
$$

Thus we get

$$
\int_{K} f d M=\int_{B}\left[\underline{\int_{H \cdot m} f(h \cdot m) d \dot{h}}\right] \delta(\pi(m)) d B(\pi(m)) .
$$

The normal component of the bundle-like metric $d s^{2}=g_{i j}(x, y) w^{i} w^{j}+$ $g_{\alpha \beta}(y) d y^{\alpha} d y^{\beta}$ is $d s_{N}^{2}=g_{\alpha \beta}(y) d y^{\alpha} d y^{\beta}$, thus $L_{B}$ is defined by the LeviCivita connection associated with the metric defined from $d S_{N}^{2}$. Thus we observe that

$$
\Delta\left(L_{D}\right)=L_{B}+\text { lower order terms . }
$$


The operator $L_{D}$ restricted to $\mathscr{E}_{S}^{0}(M)$ is symmetric with respect to $d M$ (cf. [8]), that is,

$$
\int_{M} L_{D}\left(f_{1}\right) f_{2} d M=\int_{M} f_{1} L_{D}\left(f_{2}\right) d M
$$

for $f_{1}, f_{2} \in \mathscr{E}_{S}^{0}(M)$.

For $f \in \mathscr{C}_{s}(M)$ and $m \in M$, we get

$$
\int_{H \cdot m} f d \dot{h}=\underline{f}(\pi(m)) c
$$

where $c$ denotes a nonzero constant $\int_{H \cdot m} d \dot{h}$. Putting $\underline{f}_{1}=\Phi\left(f_{1}\right), \underline{f}_{2}=$ $\Phi\left(f_{2}\right)$ for $f_{1}, f_{2} \in \mathscr{E}_{S}^{0}(M)$, we get

$$
\begin{aligned}
\int_{M} L_{D}\left(f_{1}\right) f_{2} d M & =\int_{B}\left[\int_{H \cdot m} L_{D}\left(f_{1}\right) f_{2} d \dot{h}\right] \delta d B \\
& =\int_{B}\left[\int_{\underline{H \cdot m}} L_{D}\left(f_{1}\right) d \dot{h}\right] c \delta f_{2} d B \\
& =c^{2} \int_{B} \underline{L_{D}\left(f_{1}\right)} \underline{f}_{2} \delta d B .
\end{aligned}
$$

Thus we get from $\left({ }^{* * *}\right)$

$$
\int_{B} \underline{L_{D}\left(f_{1}\right) f_{2}} \delta d B=\int_{B} \underline{f_{1}} \underline{L_{D}\left(f_{2}\right)} \delta d B
$$

for $f_{1}, f_{2} \in \mathscr{E}_{S}^{0}(M)$. By the definition of $\Delta\left(L_{D}\right)$ we get $\underline{L_{D}(f)}=$ $\Delta\left(L_{D}\right)(\underline{f})$ for $f \in \mathscr{E}_{S}(M)$, so

$$
\int_{B} \Delta\left(L_{D}\right)\left(\underline{f}_{1}\right) \underline{f}_{2} \delta d B=\int_{B} \underline{f}_{1} \Delta\left(L_{D}\right)\left(\underline{f}_{2}\right) \delta d B
$$

This expression implies that $\Delta\left(L_{D}\right)$ is symmetric with respect to $\delta d B$. Since $L_{B}$ is symmetric with respect to $d B, \delta^{-1 / 2} L_{B} \circ \delta^{1 / 2}$ is symmetric with respect to $\delta d B$ and it clearly agrees with $L_{B}$ up to lower order terms. The symmetric operators $\Delta\left(L_{D}\right)$ and $\delta^{-1 / 2} L_{B} \circ \delta^{1 / 2}$ agree up to an operator of order $\leqq 1$, thus this operator, being symmetric, must be a function. By applying the operators to the constant function 1 , we get

$$
\Delta\left(L_{D}\right)(1)-\delta^{-1 / 2} L_{B} \circ \delta^{1 / 2}(1)=-\delta^{-1 / 2} L_{B}\left(\delta^{1 / 2}\right) .
$$

Thus

$$
\Delta\left(L_{D}\right)=\delta^{-1 / 2} L_{B} \circ \delta^{1 / 2}-\delta^{-1 / 2} L_{B}\left(\delta^{1 / 2}\right) .
$$

This completes the proof of our theorem.

REMARK. The example of " $R S$-manifold of almost fibered type" 
given by $S$. Kashiwabara (Apendix 5 in [3]) is a foliated manifold with a 1-dimensional foliation and bundle-like metric. Each leaf of the foliation is a "S-geodesic." This example is constructed from the space $D$ which consists of all points $x_{1} e_{1}+x_{2} e_{2}+x_{3} e_{3}+t e_{4}$ such that $\left|x_{i}\right| \leqq 1(i=1,2,3), 0 \leqq t \leqq 1$, where $\left(e_{1}, e_{2}, e_{3}, e_{4}\right)$ denotes an orthonormal frame with origin $o$ in Euclidean 4 -space. If $S$-geodesics are of direction of $e_{4}$, a leaf through the origin $o$ has nontrivial holonomy group. Then $\delta=1$.

REMARK. The semi-reducible Riemannian space are a special class of foliated manifolds with bundle-like metrices. The metric of such a space has the local expression

$$
d s^{2}=\sigma(y) q_{i j}(x) d x^{i} d x^{j}+g_{\alpha \beta}(y) d y^{\alpha} d y^{\beta}
$$

(cf. [4]). Then $\delta$ is defined from $\sigma$.

\section{REFERENCES}

1. W. J. Baily, Jr., The decomposition theorem for V-manifolds, Amer. J. Math., 78 (1956), 862-888.

2. S. Helgason, A formula for the radial part of the Laplace-Beltrami operator, J. Differential Geometry, 6 (1972), 411-419.

3. S. Kashiwabara, The structure of a Riemannian manifold admitting a parallel field of one-dimensional tangent vector subspaces, Tohoku Math. J., 11 (1959) 327-350.

4. B. L. Reinhart, Foliated manifolds with bundle-like metrices, Ann. of Math., 66 (1959), 119-132.

5. — Closed metric foliations, Michigan Math. J., 8 (1961), 7-9.

6. I. Satake, On generalization of the notion of manifolds, Proc. Nat. Acad. Sci. U. S. A., 42 (1956), 359-363.

7. - The Gauss-Bonnet theorem for V-manifolds, J. Math. Soc. of Japan, 9 (1957), 464-492.

8. I. Vaisman, Cohomology and Differential Forms, Marcel Dekker, Inc., New York, 1973.

Received July 6, 1976 and in revised form October, 25, 1976.

KANAZAWA UNIVERSITY

MaRUNOUChI, Kanazawa 920, JapaN 


\section{PACIFIC JOURNAL OF MATHEMATICS}

\section{EDITORS}

RICHARD ARENS (Managing Editor)

University of California

Los Angeles, CA 90024

R. A. BEAUMONT

University of Washington

Seattle, WA 98105

C. C. Moore

University of California

Berkeley, CA 94720

\section{J. DugundJI}

Department of Mathematics

University of Southern California

Los Angeles, CA 90007

R. Finn and J. Milgram

Stanford University

Stanford, CA 94305

\section{ASSOCIATE EDITORS}

\section{E. F. BECKENBACH}

B. H. NEUMANN

F. WoLF

K. YOSHIDA

\section{SUPPORTING INSTITUTIONS}

\author{
UNIVERSITY OF BRITISH COLUMBIA \\ CALIFORNIA INSTITUTE OF TECHNOLOGY \\ UNIVERSITY OF CALIFORNIA \\ MONTANA STATE UNIVERSITY \\ UNIVERSITY OF NEVADA \\ NEW MEXICO STATE UNIVERSITY \\ OREGON STATE UNIVERSITY \\ UNIVERSITY OF OREGON \\ OSAKA UNIVERSITY
}

\author{
UNIVERSITY OF SOUTHERN CALIFORNIA \\ STANFORD UNIVERSITY \\ UNIVERSITY OF HAWAII \\ UNIVERSITY OF TOKYO \\ UNIVERSITY OF UTAH \\ WASHINGTON STATE UNIVERSITY \\ UNIVERSITY OF WASHINGTON \\ AMERICAN MATHEMATICAL SOCIETY
}

The Supporting Institutions listed above contribute to the cost of publication of this Journal, but they are not owners or publishers and have no responsibility for its content or policies.

Mathematical papers intended for publication in the Pacific Jaurnal of Mathematics should be in typed form or offset-reproduced, (not dittoed), double spaced with large margins. Please do not use built up fractions in the text of your manuscript. You may however, use them in the displayed equations. Underline Greek letters in red, German in green, and script in blue. The first paragraph or two must be capable of being used separately as a synopsis of the entire paper. Items of the bibliography should not be cited there unless absolutely necessary, in which case they must be identified by author and Journal, rather than by item number. Manuscripts, in triplicate, may be sent to any one of the editors. Please classify according to the scheme of Math. Reviews, Index to Vol. 39. All other communications should be addressed to the managing editor, or Elaine Barth, University of California, Los Angeles, California, 90024.

The Pacific Journal of Mathematics expects the author's institution to pay page charges, and reserves the right to delay publication for nonpayment of charges in case of financial emergency

100 reprints are provided free for each article, only if page charges have been substantially paid. Additional copies may be obtained at cost in multiples of 50 .

The Pacific Journal of Mathematics is issued monthly as of January 1966. Regular subscription rate: $\$ 7200$ a year (6 Vols., 12 issues). Special rate: $\$ 36.00$ a year to individual members of supporting institutions.

Subscriptions, orders for back numbers, and changes of address should be sent to Pacific Journal of Mathematics, 103 Highland Boulevard, Berkeley, California, 94708.

PUBLISHED BY PACIFIC JOURNAL OF MATHEMATICS, A NON-PROFIT CORPORATION

Printed at Kokusai Bunken Insatsusha (International Academic Printing Co., Ltd.). 8-8, 3-chome, Takadanobaba, Shinjuku-ku, Tokyo 160, Japan.

Copyrit (C) 1975 by Pacific Journal of Mathematics Manufactured and first issued in Japan 


\section{Pacific Journal of Mathematics \\ Vol. 69, No. $2 \quad$ June, 1977}

Carol Alf and Thomas Alfonso O'Connor, Unimodality of the Lévy spectral

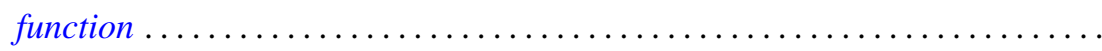

S. J. Bernau and Howard E. Lacey, Bicontractive projections and reordering of

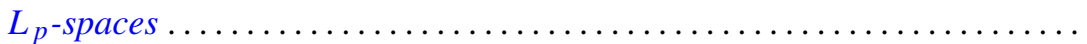

Andrew J. Berner, Products of compact spaces with bi-k and related spaces..... 303

Stephen Richard Bernfeld, The extendability and uniqueness of solutions of ordinary differential equations ...............................

Marilyn Breen, Decompositions for nonclosed planar m-convex sets ..........

Robert F. Brown, Cohomology of homomorphisms of Lie algebras and Lie

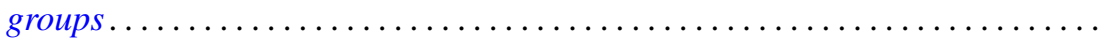

Jack Douglas Bryant and Thomas Francis McCabe, A note on Edelstein's iterative test and spaces of continuous functions ....................

Victor P. Camillo, Modules whose quotients have finite Goldie dimension ....... 333

David Downing and William A. Kirk, A generalization of Caristi's theorem with

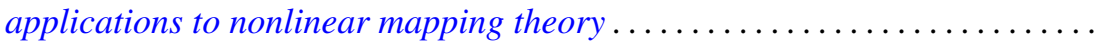

Daniel Reuven Farkas and Robert L. Snider, Noetherian fixed rings ...........

Alessandro Figà-Talamanca, Positive definite functions which vanish at

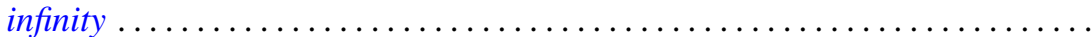

Josip Globevnik, The range of analytic extensions .................. 365

André Goldman, Mesures cylindriques, mesures vectorielles et questions de

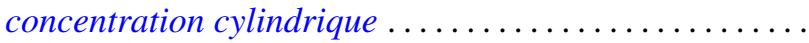

Richard Grassl, Multisectioned partitions of integers..........

Haruo Kitahara and Shinsuke Yorozu, A formula for the normal part of the

Laplace-Beltrami operator on the foliated manifold .... .

Marvin J. Kohn, Summability $R_{r}$ for double series .........

Charles Philip Lanski, Lie ideals and derivations in rings with involution ..

Solomon Leader, A topological characterization of Banach contractions . .

Daniel Francis Xavier O’Reilly, Cobordism classes of fiber bundles . .

James William Pendergrass, The Schur subgroup of the Brauer group . .

Howard Lewis Penn, Inner-outer factorization of functions whose Fourier series

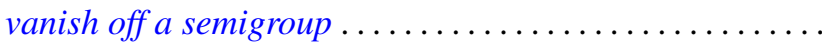

501

William T. Reid, Some results on the Floquet theory for disconjugate definite

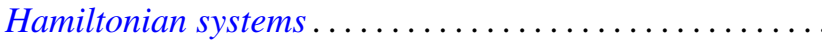

Caroll Vernon Riecke, Complementation in the lattice of convergence

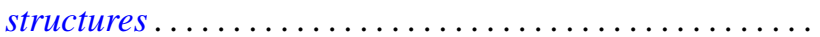

Louis Halle Rowen, Classes of rings torsion-free over their centers ......... 527

Manda Butchi Suryanarayana, A Sobolev space and a Darboux problem ....... 535

Charles Thomas Tucker, II, Riesz homomorphisms and positive linear maps.... 551

William W. Williams, Semigroups with identity on Peano continua ........... 557

Yukinobu Yajima, On spaces which have a closure-preserving cover by finite 